\title{
Spectrum Assignment with Non-deterministic Bandwidth of Spectrum Holein Cognitive Radio Networks
}

\author{
Huang Jie , He Yuan, Zeng Xiaoping, Tan Xiaoheng and Miao Lijuan \\ College of Communication Engineering, Chongqing University, Chongqing, China
}

\begin{abstract}
The spectrum allocation for cognitive radio networks (CRNs) has received considerable studies under the assumption that the bandwidth of spectrum holes is static. However, in practice, the bandwidth of spectrum holes is timevaried due to primary user/secondary user (PU/SU) activity and mobility, which result in non-determinacy. This paper studies the spectrum allocation for CRNs with non-deterministic bandwidth of spectrum holes. We present a novel probability density function (PDF) model through order statistic to describe the non-deterministic bandwidth of spectrum holes and provide a bound to approximate it. After that, a statistical spectrum allocation model based on stochastic multiple knapsack problem (MKP) is established for spectrum allocation with non-deterministic bandwidth of spectrum holes. To reduce the computational complexity, we transform this stochastic programming probleminto a constant MKP though exploiting the properties of cumulative distribution function (CDF), which can be solved via MTHG algorithm by using auxiliary variable. Simulation results illustrate that the proposed statistical spectrum allocation algorithm can achieve better performances compared to the existing algorithms when the bandwidth of spectrum holes istime-varied.
\end{abstract}

Keywords-cognitive radio; time-varied; spectrum allocation; non-deterministic bandwidth of spectrum holes; stochastic programming.

\section{Introduction}

Radio spectrum is becoming one of the most important and scarcest resources in wireless communication system. However, because of the current spectrum allocation policies, the spectrum utilization efficiency in licensed spectrum is very low [1], which generates many non-continuous vacant frequency bands, referred to as spectrum holes [2]. Cognitive radio (CR) which allows secondary users (SUs) to opportunistically utilize the frequency spectrum originally assigned to licensed primary users (PUs) is a promising approach to alleviate spectrum scarcity.

Considerable achievementhave been made for spectrum allocation in CRNs by adopting game theory [3]-[4], graph theory[5]-[6] and linear programming[7]-[9], which assume that the parameters of radio environment are static. However, in practice, due to the imperfect spectrum sensing, transmission delay and time-varied spectrum environment etc, it is difficultforthe secondary network to have theaccuratereal-timeparametersin CRNs, which will make the parameters non-deterministic. Therefore, in fact, static spectrum allocationalgorithm without considering non- deterministic parameters may result in frequent spectrum collision and poor performance. Recently, dynamic resource allocation with non-deterministic parameters in CRNs has received considerable interest from academia, which mainly focus on non-deterministic channel gain and mutual interference [10]-[12]. [10] studies the resource allocation for CRNs under non-deterministic signal-to-interference-plusnoise ratio (SINR) and propose a power control scheme by using water filling algorithm and stochastic programming. On the other hand, [11] studies the distributed resourceallocation problem in CRNs by considering the nondeterministic channel gainand the authors propose arobustdistributedpowercontrol algorithm by applying secondorder cone programming (SOCP).[12] proposes a robust distributed uplink power allocation algorithm by using worst case robust optimization method, which consider channel gains from SUs to PUs' base station, and interferencecaused by PUs to the SUs' base station are nondeterministic. Inaddition,most existing works mainly focus on non-deterministic channel gain and mutual interference. However, due to PU/SU activity and mobility, spectrum environment will change frequently, which result in time- 
variedbandwidth of spectrum holes. Therefore, exploring a spectrum allocation algorithm by considering nondeterministic bandwidth of spectrum holes is necessary for future CRNs, which will reduce spectrum collision and greatlyimprove spectrum efficiency. To the best ofour knowledge, non-continuous spectrum allocation in CRNs by considering non-deterministic bandwidth of spectrum holeshas not beenstudied in previous works.

This paper studies the non-continuous spectrum allocation problem in CRNs where the bandwidth of spectrum holes isnon-deterministic due to PU/SU activity and mobility. We present a novel PDF model through order statistic to describe the non-deterministic bandwidth of spectrum holes and provide a bound to approximate it. This PDF model is different from existing researches which mainly focus on the time duration of spectrum holes[13][14]. After that, a statistical spectrum allocation model based on stochastic MKP is established for spectrum allocation with non-deterministic bandwidth of spectrum holes. To reduce the computational complexity, we transform this stochastic programming probleminto a constant MKP though exploiting the properties of $\mathrm{CDF}$, which can be solved via MTHG algorithm by using auxiliary variable. Simulation results illustrate that the proposed statistical spectrum allocation algorithm can achieve better performances compared to the existing algorithms when the bandwidth of spectrum holes istime-varied.

The remainder of this paper is organized as follows. The layout problem is inSection II.InSection III, the statistical model for bandwidth of spectrum holes is presented. In Section IV, the statistical spectrum allocation algorithm based on stochastic MKP methodisproposed. Numerical results are provided in Section $\mathrm{V}$ to demonstrate theadvantages of the proposed schemes. We conclude this paper inSection VI.

\section{Layout of Problem}

In this CRNs system, the spectrum occupancy over frequency domain at a certain time is non-continuous, as depicted in Fig. 1 The totally frequency band is $T$, and the number of spectrum holes is $n . x(i)$ and $y(i)$ are used to denote the start point of spectrum occupancy and the occupied bandwidth. The bandwidth of spectrum hole are denoted by $b$. To describe the non-deterministic bandwidth of spectrum holes, we establish a PDF model for bandwidth of spectrum holes and based on this, a statistical spectrum allocation algorithm is proposed.

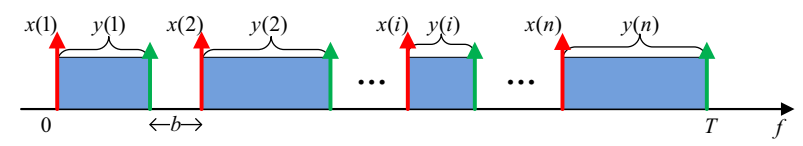

Figure 1. Spectrum occupancy over frequency domain
3. Statistical
Modelfor ofSpectrumHoles

In this section, the non-deterministic bandwidth of spectrum holes has been formulated in a mathematic way. We consider the bandwidth of spectrum holes as a part of interval between two adjacent order statistics and represent it through a random variable. Then, base on the properties of two adjacent order statistics, the PDF of bandwidth of spectrum holes can be derived.

Theorem 1: The PDF of bandwidth of spectrum holes when $x(i)$ follows a uniform distribution $U(0, T)$ and $y(i)$ follows a conditional uniform distribution $U_{y}(0, x(i+1)-x(i))$ is given by

$$
f_{b}(b)=\lambda E_{1}(\lambda b)(1)
$$

Where $\lambda=\frac{n}{T}$, and the upper and lower bounds for $f_{b}(b)$ is

$$
\frac{1}{2} \lambda e^{-\lambda b} \ln \left(1+\frac{2}{\lambda b}\right)<f_{b}(b)<\lambda \mathrm{e}^{-\lambda b} \ln \left(1+\frac{1}{\lambda b}\right)
$$

(This derivation method can also be used when $x(i)$ and $y(i)$ follow other distributions)

Proof: Let $z(i)=x(i+1)-x(i)$, where $z(i)$ represents the interval between two adjacent order statistics. Then the bandwidth of spectrum holes can be achieved by $b(i)=z(i)-y(i)$. According to [15], the joint probability distribution function of two order statistics can be obtained as

$$
\begin{aligned}
f_{x(i), x(j)}(u, v)= & \frac{n !}{(i-1) !(j-1-i) !(n-j) !} f_{x}(u) . \\
& f_{x}(v)\left[F_{x}(u)\right]^{i-1}\left[F_{x}(v)-F_{x}(u)\right]^{j-1-i} . \\
& {\left[1-F_{x}(v)\right]^{n-j} \quad-\infty<u<v<\infty }
\end{aligned}
$$

When $x(i)$ follows uniform distribution, $f_{x}(x)=\frac{1}{T}$, $F_{x}(x)=\frac{x}{T}$, the probability distribution function $z$ can be expressed as

$$
\begin{aligned}
f_{z}(z) & =\int_{-\infty}^{+\infty} f_{x(i), x(i+1)}(u, u+z) d u \\
& =\frac{1}{T} \frac{n !}{(i-1) !(n-i-1) !}\left(1-\frac{z}{T}\right)^{n-1} \int_{0}^{1} t^{i-1}(1-t)^{n-i-1} d t
\end{aligned}
$$

Substituting

$$
B(\alpha, \beta)=\int_{0}^{1} u^{\alpha-1}(1-u)^{\beta-1} d u
$$

$B(\alpha, \beta)=\frac{\Gamma(\alpha) \Gamma(\beta)}{\Gamma(\alpha+\beta)}$ in (4) yields

$$
f_{z}(z)=\frac{n}{T}\left(1-\frac{z}{T}\right)^{n-1} \approx \frac{n}{T} e^{-\frac{n}{T} z}
$$


Since $y(i)$ follows a conditional uniform distribution, uniformly distributed in $(0, z)$, according to Bayes' theorem, the joint probability distribution function $f_{y, z}(y, z)$ can be obtain as

$$
f_{y, z}(y, z)=\frac{1}{z} \cdot \frac{n}{T} e^{-\frac{n}{T} z}
$$

The probability distribution function of $b$ can be given as

$$
f_{b}(b)=\int_{-\infty}^{+\infty} f_{y, z}(y, y+b) d y=\frac{n}{T} \int_{b}^{T} \frac{e^{-\frac{n}{T} u}}{u} d u(7)
$$

Substitutingexponential integral function, $E_{1}(a x)=\int_{x}^{\infty} \frac{e^{-a x}}{x} d x$, in $(7)$, result in

$$
f_{b}(b)=\lambda\left[\mathrm{E}_{1}(\lambda b)-\mathrm{E}_{1}(n)\right]
$$

Let $\lambda=\frac{n}{T}$, since the value of $\mathrm{E}_{1}(n)$ is minimal, (8) can be approximated as $\lambda E_{1}(\lambda b)$. Based on the properties of exponential integralfunction[16], we obtain the upper and lower bounds for $f_{b}(b)$ is given as

$$
\frac{1}{2} \lambda e^{-\lambda b} \ln \left(1+\frac{2}{\lambda b}\right)<f_{b}(b)<\lambda \mathrm{e}^{-\lambda b} \ln \left(1+\frac{1}{\lambda b}\right)
$$

\section{StatisticalSpectrumAllocationAlgorith mBased on StochasticMKP}

\subsection{Spectrum allocation model based on stochastic MKP}

We assume that the CRNs consist of $m$ SUs and $n$ spectrum holes, where SUs'data rate requirements and the bandwidth of spectrum holes are indicated as $R=\left\{r_{i}, \ldots, r_{m}\right\}$ and $C=\left\{c_{1}, \ldots, c_{n}\right\}$ respectively. Among this, each SU $i$ $(i=1,2,3, \cdots, m)$ can utilize any spectrum hole $j$ $(j=1,2,3, \cdots, n)$, which means the non-continuous spectrum allocation problem can be transformed into a kind of MKP to achieve maximum transmission rate.

$$
\begin{gathered}
\text { maximize } z=\sum_{i=1}^{m} \sum_{j=1}^{n} r_{i} x_{i, j}(10) \\
\text { s.t. } \sum_{i=1}^{m} b_{i} \cdot x_{i, j} \leq c_{j} \quad j \in N=\{1, \ldots, n\}(11) \\
\sum_{j=1}^{n} x_{i, j} \leq 1 \quad i \in M=\{1, \ldots, m\}(12) \\
x_{i, j}=0 \text { or } 1 \quad i \in M \quad j \in N(13)
\end{gathered}
$$

Let $b_{i}$ be the necessary bandwidth that satisfieddata rate requirement $r_{i}$ and $x_{i, j}=1$ represent $\mathrm{SU} i$ can allocated into spectrum hole $j$. Moreover, by adopting AMC scheme, SUs with different positions can achieve different data rate in same channel due to environment differences. According to G.J.Foschini's inference[17], the bandwidth needs for SU $i$ on spectrum hole $j$ can be given as

$$
b_{i, j}=\frac{r_{i}}{\log _{2}\left(1+\frac{\gamma_{i, j}}{-\frac{2}{3} \ln \frac{P_{b}}{2}}\right)}
$$

Where $P_{b}$ denotes maximum tolerable error rate and $\gamma_{i, j}$ indicates the SINR when SU $i$ transmits on spectrum hole $j$. Substituting (14) into (11), we obtain a special MKP model, where each SU requires different bandwidth when allocated into different spectrum holes. Then (11) can be expressed as

$$
\sum_{i=1}^{m} \frac{b_{i, j}}{c a_{B}} \cdot x_{i, j} \leq \frac{c_{j}}{c h_{B}} \cdot c a_{n u m} \quad j \in N=\{1, \ldots, n\}
$$

Where $c a_{B}$ is the subcarrier bandwidth, $c h_{B}$ denotes the channel bandwidth, $c a_{\text {num }}$ indicates the number of subcarriers in each channel.

To consider non-deterministic bandwidth of spectrum holes, we take previous statistical model into this MKP model. Let $c_{j, t}$ be the available bandwidth of spectrum hole $j$ detected bySUs' base station at time $t$ and $C_{j}=\left\{c_{j, t-k+1}, c_{j, t-k+2} \ldots c_{j, t}\right\} \quad$ represents the record of bandwidth of spectrum hole $j$ in recent $k$ times spectrum sensing. We can determine the parameter $\lambda$ of the statistical model by applying point estimation method. The expectation of previous PDF can be written as

$$
E(b)=\int_{0}^{\infty} \lambda b E_{1}(\lambda b) d b=-\lambda \cdot\left(-\frac{\Gamma(2)}{2 \lambda^{2}}\right)=\frac{1}{2 \lambda}(16)
$$

Hence, based on the method of moment[15], the PDF parameter $\lambda_{j}$ of bandwidth of spectrum hole $j$ can express as $1 / 2 E\left(C_{j}\right)$.

We assume $c_{j}$ is a random variable in (15) and follows the PDF (1), which transmits the MKP spectrum allocation model above into a stochastic programming model. The unknown random variable introduces uncertainty to (15) and makes the constraint quit complicated. Thus, to reduce the complexity, we tackle this issue by using chance-constraint programming method which finds the optimum solution under the condition that the constraints is feasible with probability greater than the threshold $\alpha$. The constraint (15) above can be rewritten as

$$
\text { s.t. } \operatorname{Pr}\left\{\sum_{i=1}^{m} \frac{b_{i, j}}{c a_{B}} \cdot x_{i, j} \leq \frac{c_{j}}{c h_{B}} \cdot c a_{\text {num }}\right\} \geq \alpha_{j} \quad j \in N(17)
$$


This indicates the constraint must be feasible with the probability greater than $\alpha_{j}$ and this model maximizes the transmission rate by satisfying the constraint with high probability.

\subsection{Simplification and solution}

To reduce the computational complexity, we simplify the stochastic programming model above by the usage of the properties of CDF. For (17), since $c_{j}$ follows the $\mathrm{CDF}$ $F_{j}(c)$, there must exist a $K_{j}$ for each threshold $\alpha_{j}$ $\left(0 \leq \alpha_{j} \leq 1\right)$ satisfies $\operatorname{Pr}\left\{K_{j} \leq c_{j}\right\}=\alpha_{j}$. Therefore, (17) will be satisfied only if

$$
\sum_{i=1}^{m} \frac{b_{i, j} \cdot c h_{B}}{c a_{B} \cdot c a_{n u m}} \cdot x_{i, j} \leq K_{j}
$$

Furthermore, as $\operatorname{Pr}\left\{K_{j} \leq c_{j}\right\}=1-F_{j}\left(K_{j}\right), K_{j}$ can be represented by $F_{j}^{-1}\left(1-\alpha_{j}\right)$. We can transmit (17) as

$$
\sum_{i=1}^{m} \frac{b_{i, j} \cdot c h_{B}}{c a_{B} \cdot c a_{n u m}} \cdot x_{i, j} \leq F_{j}^{-1}\left(1-\alpha_{j}\right) \quad j \in N=\{1, \ldots, n\}
$$

Since the model proposed is a NP-hard problem, the existing algorithm obtains the solution by exhaustive search which will encounter complex computationswhen $n$ and $m$ have large values. Hence, we convert the model into a Generalized Assignment Problem (GAP) by using auxiliary variable and solve it via MTHG algorithm[17] which will get the suboptimal solution. Let

$$
\frac{b_{i, j} \cdot c h_{B}}{c a_{B} \cdot c a_{\text {num }}}=w_{i, j}, \tilde{c}_{j}=F_{j}^{-1}\left(1-\alpha_{j}\right), p_{i, j}=r_{i}, j \in N
$$

and adding auxiliaryvariables $p_{i, n+1}=0, \quad \tilde{c}_{n+1}=m$ and $w_{i, n+1}=1, i \in M$. Then, the model can be rewritten as

$$
\begin{gathered}
\text { maximize } \quad z=\sum_{i=1}^{m} \sum_{j=1}^{n} p_{i, j} x_{i, j} \quad \text { (20) } \\
\text { s.t. } \sum_{i=1}^{m} w_{i, j} \cdot x_{i, j} \leq \tilde{c}_{j} \quad j \in \tilde{N}=\{1, \ldots, n, n+1\} \\
\sum_{j=1}^{n} x_{i, j}=1 \quad i \in M=\{1, \ldots, m\} \\
x_{i, j}=0 \text { or } 1 \quad i \in M \quad j \in \tilde{N}
\end{gathered}
$$

This GAP model means each SU must be allocated in a unique $\tilde{c}_{j}$ and $\tilde{c}_{n+1}$ contains the SUs which have not be allocated due to lack of spectrum resource. The GAP model can be solved by using MTHG algorithm, where iteratively consider all the unassigned SUs, and assign the SU $i^{*}$ having the maximum difference between the largest and second largest $p_{i, j}$ to the spectrum hole $j^{*}$ for which $p_{i^{*}, j^{*}}$ is a $\operatorname{maximum}\left(p_{i^{*}, j^{*}}=\max \left\{p_{i, j}: j \in \tilde{N}\right\}\right)$.

\section{Simulation Results}

In this section, the performance of proposed statistical spectrum allocation algorithm has be studied
thoughnumericalsimulations.We assume that all SUslocated around SUs' base station with the distance randomly ranged from 50 to $2000 \mathrm{~m}$ and the simulation parameters are set as TableI. In order to simulate the time-variedbandwidth of spectrum holes caused by PU/SU activity and mobility, we randomly generate spectrum holes with the bandwidth changed through change rate $p$ (the probability of bandwidth of spectrum hole changed next moment) over the frequency band $W$. Then, we compare the performance of proposed statistical MKP spectrum allocation algorithm with static MKP spectrum allocation algorithm in [8].

TABLE I. SIMULATIONPARAMETERS

\begin{tabular}{ll}
\hline Parameters & Values \\
\hline SU output power $P_{\max }$ & $45 \mathrm{dBm}$ \\
Maximum tolerable error rate $P_{b}$ & $10^{-6}$ \\
SU number $S M U N$ & 30 \\
Spectrum hole number $S H$ & $3 \sim 6$ \\
Noise power $N_{0} B$ & $-111 \mathrm{dBm}$ \\
Chance programming threshold $\alpha$ & 0.5 \\
Bandwidth of spectrum hole change rate $p$ & $0.1 \sim 1$ \\
Total frequency band $W$ & $10 \mathrm{MHZ}$
\end{tabular}

Fig. 2illustrates the CDF of SU spectrum efficiency using the two algorithmswith different situation ( $p=0.5$ and $p=1)$. As shown in Fig. 2, statistical MKP algorithm and static MKP algorithm occupy $61 \%$ and $52 \%$ respectively when the spectrum efficiency is large than 1.8 , which means statistical MKPalgorithm has larger percentage of high spectrum efficiency than the static MKP algorithm. This case is becausestatistical MKP algorithm which takes the statistical properties of bandwidth of spectrum holes into consideration can achieve dynamic optimal and reduce bandwidth collisions caused by spectrum holes changes. Moreover, as $p$ increased to 1, statistical MKP algorithm exceeds static MKP algorithm more, which means statistical MKP algorithm can better adapt to the scenario with frequency time-varied bandwidth of spectrum holes and achieve high spectrum efficiency.

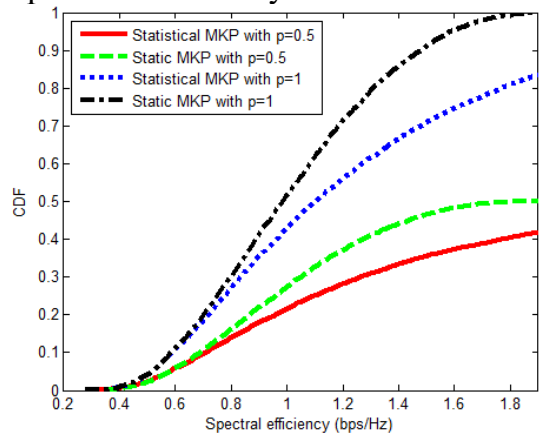

Figure 2. CDF of spectrum efficiency 
In Fig.3, we evaluate the network utility with different bandwidth change rate $p$. The network utility is defined as[18]

$$
U=\sum_{k=1}^{m} \ln R(\mathrm{k})(24)
$$

Where $R(\mathrm{k})$ is the throughput for SU $\mathrm{k}$. In theFig. 3, as $p$ increased, the network utility is reduced due to bandwidth collision. The static MKP algorithm suffers lower networkutility thanstatistical MKP algorithm, because it only considers current static optimal and use constant allocation parameters, which may lead to more bandwidth collision. In addition, when the number of spectrum holes increase, the network utility of statistical MKP algorithm increase more than the static MKP algorithm, which means statistical MKP algorithm can obtain a good performance under the condition of non-continuous spectrum.

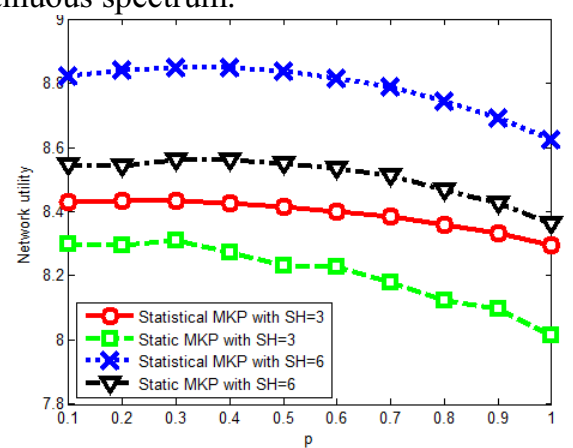

Figure 3. Network utility with different bandwidth of spectrum holes change rate $p$

Fig. 4 shows the average spectrum collision rate with different bandwidth change rate $p$. From Fig. 4 , we can see that as $p$ increase, the spectrum collision rate increased dramatically and statistical MKP algorithm achieve lower collision rate than the static MKP algorithm. It is becausestatistical MKP algorithm can relax the bandwidth collision through assigning spectrum resource with considering the statistical properties of spectrum holes. The result shows that statistical MKP algorithm can effectively reduce the spectrum collision when spectrum holes changes.

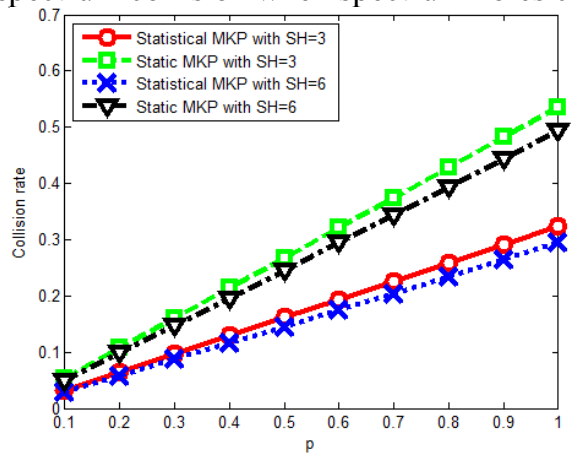

Figure 4. Average spectrum collision rate with different bandwidth of spectrum holes change rate $p$

\section{Conclusion}

In this paper, we study the problem of non-continuous spectrum allocation in CRNs where the bandwidth of spectrum holes is non-deterministic due to PU/SU activity and mobility. We present a novel PDF model through order statistic to describe the non-deterministic bandwidth of spectrum holes and provide a bound to approximate it.After that, a statistical spectrum allocation model based on stochastic MKP is established for spectrum allocation with non-deterministic bandwidth of spectrum holes. To reduce the computational complexity, we transform this stochastic programming probleminto a constant MKP though exploiting the properties of CDF, which can be solved via MTHG algorithm by using auxiliary variable. Simulation resultsverify that the proposedstatistical spectrum allocation algorithm can achieve better performances compared to the existing algorithms when the bandwidth of spectrum holes istime-varied.

\section{Acknowledgment}

ThisworkwassupportedbytheTrainingProgramoftheMajor ResearchPlanoftheNaturalScienceFoundationofChina(Grant No.91438104)andtheGraduateStudentsResearchProgramofC hongqing(GrantNo.CYS14005).

\section{References}

1. C. Kim, C. Leem, S. Kang,"Policy and technology of dynamic spectrum access in Korea,"2008 3rd International Conference on Cognitive Radio Oriented Wireless Networks and Communications, pp. 1-4, 2008, doi: 10.1109/CROWNCOM.2008.4562456.

2. M. Mchenry,E.Livsics,T.Nguyen, "XG dynamic spectrum access field test results," IEEE Communication Magazine,pp. 51-57,Jun. 2007.

3. J. Revathy,M. Senthil,"Resource allocation in next generation networks using game theory,"2014 International Conference on Information Communication and Embedded Systems (ICICES), pp. $1-4$.

4. Z. Wu, P. Cheng, X. Wang, “Cooperative spectrum allocation for cognitive radio network: an evolutionary approach,"2011 IEEE International Conference on Communications (ICC), pp. 1-5.

5. Y. Li, L. Zhao, C. Wang,"'Aggregation-based spectrum allocation algorithm in cognitive radio networks,"Network Operations and Management Symposium (NOMS), IEEE, pp. 506-509.

6. A. Plummer, S. Biswas, "Distributed spectrum assignment for cognitive networks with heterogeneous spectrum opportunities," Wireless Communications and Mobile Computing, pp. 1239-1253, Sep. 2011.

7. F. Wu, Y. Mao, S. Leng, "A carrier aggregation based resource allocation scheme for pervasive wireless networks,"2011 IEEE Ninth International Conference on Dependable, Autonomic and Secure Computing (DASC), IEEE, pp. 196-201.

8. C. Li, W. Liu, Q. Liu, "Spectrum aggregation based spectrum allocation for cognitive radio networks,"Wireless Communications and Networking Conference (WCNC), IEEE, pp. 1626-1631.

9. Y. Song, C. $\begin{gathered}\text { Zhang, } \\ \text { multidimensional }\end{gathered}$ 
applications in cognitive radio networks,"Military Communications Conference, MILCOM. pp. 1-7.

10. P. Setoodeh, S. Haykin,"Robust transmit power control for cognitive radio," Proc. of the IEEE,vol. 97, no.5, pp. 915-939, May. 2009.

11. S. Sun, W. Ni, Y.Zhu, "Robust power control in cognitive radio networks: a distributed way,"2011 IEEE International Conference on Communications (ICC), pp. 1-6.

12. S. Parsaeefard, A. Sharafat,"Robust distributed power control in cognitive radio networks," IEEE Transactions onMobile Computing, pp. 609-620, Apr. 2013.

13. J. Misic, V. Misic,"Probability distribution of spectral hole duration in cognitive networks,"INFOCOM, 2014 Proc. IEEE.pp. 2103-2111.

14. Y. Saleem, M. Rehmani,"Primary radio user activity, models for cognitive radio networks: A survey," Journal of Network and Computer Applications, pp. 116, Mar. 2014.

15. G. Casella, R. Berger,"Statistical inference. Pacific Grove," CA: Duxbury, 2002.

16. M. Abramowitz,S. Irene, "Handbook of mathematical functions: with formulas, graphs, and mathematical tables," Courier Corporation, 1964.

17. S. Martello, P.Toth,"Knapsack problems: algorithms and computer implementations," John Wiley Sons, 1990.

18. Y. Wang, K. Pedersen, T. Sorensen,"Utility Maximization in LTE-Advanced Systems with Carrier Aggregation,"2011 IEEE 73rd Vehicular Technology Conference (VTC Spring), pp. 1 - 5. 